\title{
Lectin-Like Glycoprotein PsNLEC-1 Is Not Correctly Glycosylated and Targeted in Boron-Deficient Pea Nodules
}

\author{
Luis Bolaños, Arancha Cebrián, Miguel Redondo-Nieto, Rafael Rivilla, and Ildefonso Bonilla \\ Departamento de Biología, Facultad de Ciencias, Universidad Autónoma de Madrid, 28049-Madrid, Spain \\ Accepted 11 January 2001.
}

\begin{abstract}
Symbiosome development was studied in pea root nodules from plants growing in the absence of boron $(B)$. Rhizobia released into the host cells of nodules from B-deficient plants developed to abnormal endophytic forms with an altered electrophoretic lipopolysaccharide pattern. Immunostaining after sodium dodecyl sulfate-polyacrylamide gel electrophoresis and electroblotting of nodule homogenates with antibodies that recognize glycoprotein components showed that two previously described lectin-like glycoproteins (PsNLEC-1A and PsNLEC-1B) did not harbor the carbohydrate epitope normally recognized by specific monoclonal antibodies. Material derived from Bdeficient nodules, however, still contained three antigenic isoforms with similar electrophoretic mobilities to PsNLEC-1 isoforms A, B, and C. These could be detected following immunoblotting and immunostaining with a specific antiserum originating from the purified PSNLEC protein that had been heterologously expressed in Escherichia coli. Immunogold localization of PsNLEC-1 sugar epitopes in B-deficient nodules showed that they were associated mostly with cytoplasmic vesicles rather than normal localization in the symbiosome compartment of mature infected cells. These results suggest that a modification of the glycosyl-moieties of PSNLEC-1 and an alteration of vesicle targeting occur during the development of pea nodules in the absence of $B$, and that these changes are associated with the development of aberrant nonfunctional symbiosomes.
\end{abstract}

Boron (B), a micronutrient required in micromolar concentrations by plants, has been implicated in several metabolic pathways (Parr and Loughman 1983). These include sugar transport, cell wall synthesis, lignification, cell wall structure, carbohydrate transport, RNA metabolism, respiration, indole acetic acid metabolism, phenolics metabolism, and membrane structure and function. In addition to higher plants, B is essential for diatoms (Smyth and Dugger 1981) and heterocystous cyanobacteria (Bonilla et al. 1990). This diversity of roles played by $\mathrm{B}$ might indicate that the micronutrient is either involved in numerous processes or has a pleiotropic effect. Because of the special chemistry of $\mathrm{B}$, there is increasing evidence for the second alternative. Polyhydroxy compounds

Corresponding author: I. Bonilla; Telephone: +34 91397 8178; Fax: +34 91397 8344; E-mail: ildefonso.bonilla@uam.es with a cis-diol configuration form complexes with boric acid and/or borate anions as cross linkers (O’Neill et al. 1996). The main role suggested for $\mathrm{B}$ is related to the stabilization of precursors of glycoconjugates that account for plant metabolism (Marschner 1995).

A requirement of $\mathrm{B}$ for symbiotic $\mathrm{N}_{2}$ fixation was first suggested by Brenchley and Thornton (1925). More recently, pea nodules developed under low-B conditions have been reported to be nonfunctional and prematurely senescent (Bolaños et al. 1994). To date, studies on the role of the micronutrient in the establishment of an effective legume-Rhizobium symbiosis have indicated a $B$ requirement for nodule cell wall structure (Bonilla et al. 1997), infection thread development, and nodule invasion (Bolaños et al. 1996).

The aim of this study was to investigate symbiosome development in nodules developed under low-B conditions. Following bacterial release into the cytoplasmic space, endophytic bacteria occupying the organelle-like symbiosome continue to divide and eventually differentiate into nitrogen-fixing bacteroids (Brewin 1991). Bacteroids are enclosed by a plant-derived peribacteroid membrane (PBM) that harbors a differentiated form of plasma membrane glycocalyx composed of a mixture of glycoproteins and glycolipids (Perotto et al. 1991). Between the PBM and the bacteroid there is a peribacteroid fluid (PBF), which is probably lysosomal in character (Mellor 1989) and, in pea, contains glycoproteins, including specific nodule lectin-like glycoproteins (Kardailsky et al. 1996). During the symbiotic interaction, the structure of the symbiosome components differentiate in close synchrony (Verma 1992). Maturation implies gradual differentiation of the PBM at structural (Miao et al. 1992; Perotto et al. 1995) and functional (Day and Udvardi 1993) levels from those of the plasma membrane, targeting proteins to the PBF (Mellor 1989), and bacteroid development to a $\mathrm{N}_{2}$-fixing form (De Maagd et al. 1994; Kannenberg et al. 1994).

Several plant and bacterial glycoconjugates are implicated in this phenomenon (Kannenberg and Brewin 1994). Borate anions have the ability to become esterified with cis-diol groups from polyhydroxyl compounds (Mazurek and Perlin 1963), thus B can play a role in stabilizing glycoconjugates and/or the interactions among them during symbiosome maturation as it does in the previous steps of nodule development. We investigated the behavior of some of these glycocomponents within nodules developed in pea plants grown in the absence of the micronutrient. 


\section{RESULTS}

Ultrathin sections of nodules from pea plants growing in the presence $(+\mathrm{B})$ or absence $(-\mathrm{B})$ of $\mathrm{B}$ were analyzed by electron microscopy to examine the structure of symbiosomes (Fig. 1). In contrast to $+\mathrm{B}$ symbiosomes, which usually contain a single Y-shaped bacteroid enclosed by a peribacteroid membrane (Fig. 1A), the symbiosome-like compartment in $-\mathrm{B}$ nodules enclosed several endophytic bacteria of a smaller size that apparently did not differentiate into real bacteroids (Fig. 1B). Moreover, immunodetection of nitrogenase in nodule sections or in isolated nodule bacteria was negative in B-deficient nodules (data not shown). Figure 2 shows that the silver-stained electrophoretic profile of the lipopolysaccharide (LPS) of bacteroids derived from $+\mathrm{B}$ nodules (lane 1) did not appear in endophytic bacteria derived from nodules of $-\mathrm{B}$ treatments (lane 2), whereas no differences were found in the LPS of free-living Rhizobium leguminosarum 3,841 grown in liquid Y-minimal medium with (lane 3) or without (lane 4) B. Because changes in the silver-staining pattern of the LPS occur during bacteroid development (Sindhu et al. 1990), the abnormal LPS differentiation and the absence of detectable nitrogenase indicate that endophytic bacteria do not develop into bacteroids in pea nodules when plants are grown in the absence of B. This may be correlated with an apparent failure during the maturation of peribacteroid components.

Symbiosome development apparently involves interactions between plant-derived and bacterial cell-surface glycoconjugates, and $\mathrm{B}$ has been related to the stabilization of glycoconjugates. Therefore, components of the PBF and the glycocalyx from the PBM were analyzed with monoclonal antibodies as molecular probes to investigate the possible effects of B deprivation.

The most obvious differences during nodule development in the absence of B were found in the analysis of PBF glycoproteins by the use of MAC 254 and 266 antibodies. Because these antibodies recognize soluble glycoproteins and membrane breakage occurs in B-deficient nodules (Bolaños et al. 1994), the total soluble material obtained during fractionation of nodules was analyzed to avoid losses of symbiosomal fluid.
Immunostaining with either antibody following sodium dodecyl sulfate-polyacrylamide gel electrophoresis (SDS-PAGE) and electroblotting gave similar results (Fig. 3A). The extracts from control nodules contained antigens of a high molecular weight recognized by MAC 254. During symbiosome maturation, lower molecular weight antigens can be distinguished in the PBF (Fig. 3A). Arrowheads highlight two bands that probably correspond to the nodule-specific, lectin-like glycoproteins (PsNLEC-1A and 1B) described by Dahiya et al. (1997). In contrast to B-plus nodules (+B lanes), most antigens disappeared in B-deficient material ( $-\mathrm{B}$ lanes) during nodule development. Furthermore, the bands corresponding to PsNLEC glycoproteins were never detected.

Because MAC 254 or 266 recognize sugar epitopes, the monospecific R76 antiserum was used for PsNLEC-1 detection in order to clarify whether the lectin-like glycoproteins were absent or the carbohydrate epitopes were modified in the absence of B. Figure 3B shows that R76 recognized three bands, described previously by Dahiya et al (1997), as PsNLEC-1 isoforms A, B, and C. These isoforms apparently had a similar mobility in $+\mathrm{B}$ and $-\mathrm{B}$ treatments. Therefore, loss of MAC 254 labeling in B-deficient nodules might be attributed to the chemical modification of the glycosyl moiety of the protein.

In a parallel study of PBM glycoproteins (with MAC 207 and 272 monoclonal antibodies) and PBM glycolipids (probed with MAC 206 and 255 antibodies), no clear differences between $+\mathrm{B}$ and $-\mathrm{B}$ nodules were revealed by immunoblotting (Fig. 3C and D). Immunolocalization studies, however, indicate that most antigenic components from the PBM disappeared from the infected region in old nodules (Fig. 4), probably as a result of membrane degradation in B-deficient tissues.

Immunogold localization in ultrathin pea-nodule sections showed that antigens recognized by MAC 254 were localized in the PBF compartment of infected cells (Fig. 5A and B), which adds to earlier reports (Perotto et al. 1991). In Bdeficient nodules, however, most of the antigen was accumulated in Golgi-derived or cytoplasmic vesicles, although some labeling appeared in the normal PBF location (Fig. 5C to F). Because MAC 254 staining might be associated with several
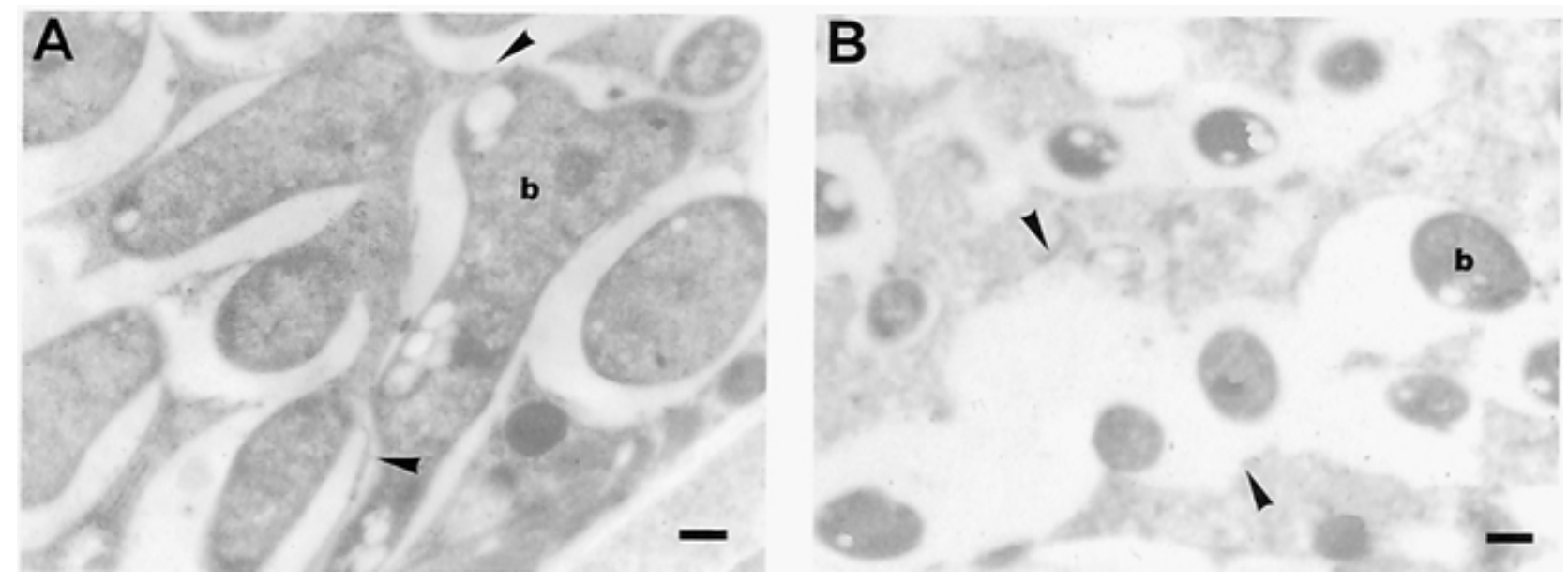

Fig. 1. Electron micrographs of pea nodule sections, 3 weeks after inoculation with Rhizobium leguminosarum 3841, from plants grown in the A, presence or $\mathbf{B}$, absence of boron. Boron deficiency leads to aberrant symbiosomes containing several bacterial cells (b) that apparently do not develop into bacteroid forms. Arrowheads show the position of the peribacteroid membrane. Bar $=0.5 \mu \mathrm{m}$. 
Golgi-derived glycoproteins carrying this epitope (Perotto et al. 1991), PsNLEC-1 was localized with the specific R76 antiserum. Localization of PsNLEC-1 by R76 staining in $+\mathrm{B}$ nodules showed that the protein was associated mostly with small inclusions bodies in the symbiosomal space resembling MAC 254 immunostaining (Fig. 6A and B). Conversely, Bdeficient nodule sections showed very little labeling in the PBF compartment and a dispersed staining associated with peribacteroid or cytoplasmic membranes (Fig. 6C and D). These observations probably indicate a failure of the targeting of the PSNLEC glycoproteins to the PBF of symbiosomes in B-deficient nodules.

\section{DISCUSSION}

During cell invasion by rhizobia and the development of $\mathrm{N}_{2}$-fixing bacteroids, there is an interesting transition in which the endophytic bacteria develop an intimate relationship with the host plant mediated by plant- and rhizobia-derived glycoconjugates, some of them apparently capable of interacting with B. In addition to the effect on the nodule cell wall structure (Bonilla et al. 1997), the absence of B has multiple effects during nodule development. The development of infection threads and the process of endocytosis are altered by the absence of the micronutrient, perhaps because the presence of borate may inhibit binding of the plant matrix glycoprotein (a component of infection threads and droplets) to the bacterial cell surface and hence promotes a closer association between this surface and the plasma membrane (Bolaños et al. 1996). Furthermore, the number of infected cells in young nodules is lower under B deficiency, as revealed by staining with MAC 206, a marker from symbiosomes (Bolaños et al. 1996). In the present study, our results show that B deficiency in pea plants leads to the development of nodule cells containing aberrant symbiosomes (Fig. 1B).

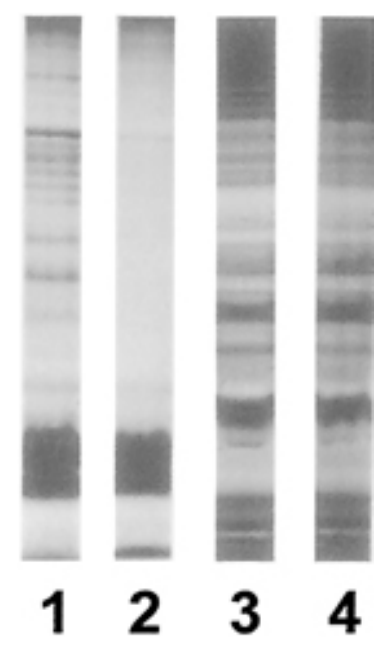

Fig. 2. Electrophoretic profiles of Rhizobium leguminosarum 3841 nodule or free-living bacteria extracts following periodate oxidation and silver staining to reveal lipopolysaccharide and other glycoconjugates. Lane 1, Bacteroids isolated from pea nodules developed in the presence of boron, 15 days postinoculation. Lane 2, Endophytic bacteria isolated from nodules developed in the absence of boron, 15 days postinoculation. Lane 3, Bacterial suspensions from liquid $\mathrm{Y}$ cultures grown in the presence of $0.1 \mu \mathrm{g}$ of $\mathrm{H}_{3} \mathrm{BO}_{3}$ per ml. Lane 4, Bacterial suspensions from liquid cultures grown in boron-free $\mathrm{Y}$ medium.
Our previous observations of abnormal bacteroid forms in B-deficient nodules were interpreted as early senescence because these nodules strongly inhibited the $\mathrm{N}_{2}$-fixing activity and show an advanced stage of membrane degradation at 3 and 4 weeks postinoculation (Bolaños et al. 1994). Although $\mathrm{B}$ is removed from the nutrient solution for pea germination and growth, a small amount can be detected (11 times lower than normal), originating from seed reserves (Bolaños et al. 1996). While B is still available, nodules can develop. Moreover, senescence is still observable for these B-deficient nodules 3 weeks postinoculation (Fig. 4), and the molecular markers used in the present study indicates that symbiosome development becomes abnormal at an earlier stage in nodules of B-deprived plants, e.g., 15 days postinoculation (Fig. 3).

Immunological analysis of peribacteroid fluid- and membrane-derived components revealed a clear effect of B deficiency on the PBF differentiation. Most of the glycoproteins recognized by MAC 254 and 266 lost reactivity with these antibodies during nodule development under B deficiency (Fig. 3A). Moreover, the 27- and 30-kDa nodule-specific lectin-like glycoproteins (Kardailsky et al 1996), which appeared during symbiosome development, were never recognized by the MAC 254 antibody (Fig. 3A). Labeling of PsNLEC by R76 (Fig. 3B) suggests that glycosylation of these proteins is altered in the absence of the micronutrient. Both $+\mathrm{B}$ and $-\mathrm{B}$ nodules expressed three bands that probably correspond with PsNLEC-1A, $\mathrm{B}$, and $\mathrm{C}$. Isoform $\mathrm{C}$ most likely carries the $\mathrm{N}$-linked heptasaccharide that is commonly associated with vacuolar glycoproteins (Faye et al. 1989), whereas isoforms A and B apparently carried a more complex side chain that is recognized by MAC 254 and 266 antibodies (N. Brewin and D. Ashford, personal communication). Although B-deficient nodules expressed R76 bands of a

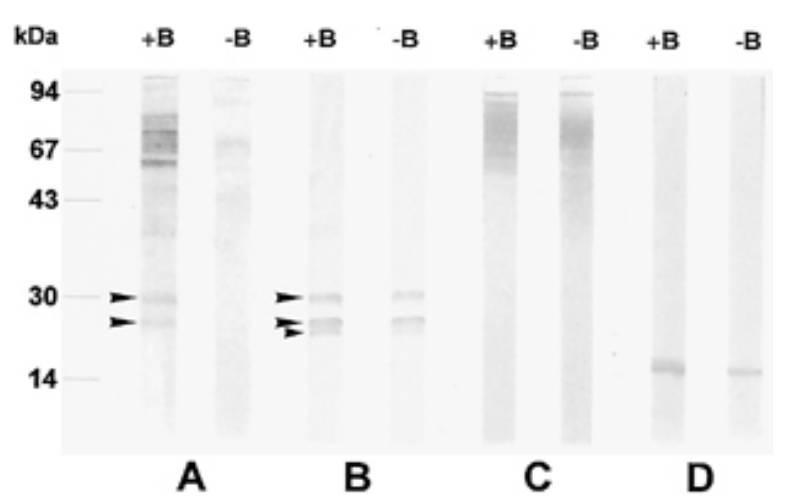

Fig. 3. Immunostaining of pea nodule-derived fractions with MAC 254 (antimembrane and anitsoluble glycoproteins) antibody with A, R76 (anti-PsNLEC-1 proteins) antiserum; B, MAC 207 (antimembrane glycoproteins) antibody; and C, MAC 206 (antimembrane glycolipids) antibody on $\mathbf{D}$, nitrocellulose membranes following sodium dodecyl sulfate-polyacrylamide gel electrophoresis and electroblotting. A and B, Soluble fractions derived from pea nodules harvested 15 days postinoculation with Rhizobium leguminosarum 3841. C and D, Membrane fractions derived from nodules harvested 21 days posinoculation. $+\mathrm{B}=$ plants grown in the presence of $0.1 \mu \mathrm{g}$ of $\mathrm{H}_{3} \mathrm{BO}_{3}$ per ml. $-\mathrm{B}=$ plants grown in the absence of added $\mathrm{H}_{3} \mathrm{BO}_{3}$. Large arrowheads indicate bands corresponding to nodule-specific, lectin-like glycoproteins (PsNLEC-1 isoforms A and B) that do not harbor MAC 254 epitopes in B deficiency. Small arrowhead shows PsNLEC-1C. Antibody binding was detected with anti-rat immunoglobulin $\mathrm{G}$ ( $\mathrm{IgG}$ ) for monoclonal antibodies or anti-rabbit IgG for R76 antiserum. Both were conjugated to alkaline phosphatase as a secondary antibody. 
similar mobility to that of PsNLEC-1A and 1B (Fig. 3B), the glycosylation must be altered because they apparently do not carry the carbohydrate epitope recognized by the MAC 254 antibody. Furthermore, in the absence of B, these glycoprotein antigens were localized mainly in cytoplasmic vesicles instead of the symbiosome compartment (Figs. 5 and 6). A role for B in the targeting of vesicles containing glycoproteins has been proposed in other plant tissues (Goldbach 1997), and accumulation of PsNLEC proteins in cytoplasmic vesicles rather than inside the symbiosome could reflect a lack of membrane fusion in $\mathrm{B}$ deficiency. Goldbach's model proposes a B-mediated bridging between hydroxylic groups (primarily mannose moieties of glycoproteins) for ligands in vesicles and membrane and the subsequent release of the vesicle contents. Because PsNLEC glycosylation is modified in $-\mathrm{B}$ nodules, the targeting of vesicles to the symbiosome compartment may be arrested as a result of the lack of B bridges and/or the absence of the proper hydroxylic ligand. There is the alternate possibility that PsNLEC-1 glycoproteins are normally targeted but suffer a rapid turnover under B-deficient conditions. Although it could be true for the carbohydrate epitopes associated with PsNLEC-1A and 1B isoforms, the data in Figure 3 seems to make this possibility unlikely.
Although the role of these lectin-like glycoproteins is still unclear, it has been hypothesized that lectins in nodules could serve as a transient storage for assimilated nitrogen (VandenBosch et al. 1994). In fact, the expression of these nodule lectin-like glycoproteins in pea nodules is stronger in the mature $\mathrm{N}_{2}$-fixing cells (Dahiya et al. 1997). The low level of PsNLEC-1 glycoprotein detected in B-deficient nodules is consistent with this hypothesis because these nodules are ineffective in nitrogen fixation. Observations made in this study, together with those from the pea-line Sprint-2 Fix $^{-}$(sym31), a symbiotically defective mutant with a very reduced expression of MAC 254 epitopes (Brewin et al. 1995; Sherrier et al., 1997), suggest that other possible roles of nodule lectin-like glycoproteins should not be ruled out. Bacteroid development appeared arrested at a juvenile stage in B-deficient nodules (Fig. 1B) and nodules of Sprint-2 Fix ${ }^{-}$(Sherrier et al. 1997; Tikhonovich et al. 1995). Nodules of sym31 that may have altered the carbon metabolism (Romanov et al. 1995) only express the fastest-migrating $(25 \mathrm{kDa})$ isoform $\mathrm{C}$ of PsNLEC1 recognized by $\mathrm{R} 76$, which is associated with nodule supernatant and cytoplasmic membrane fractions, but is absent from any symbiosomal fraction (Dahiya et al. 1998). In the present study involving B-deprivation, which also results in
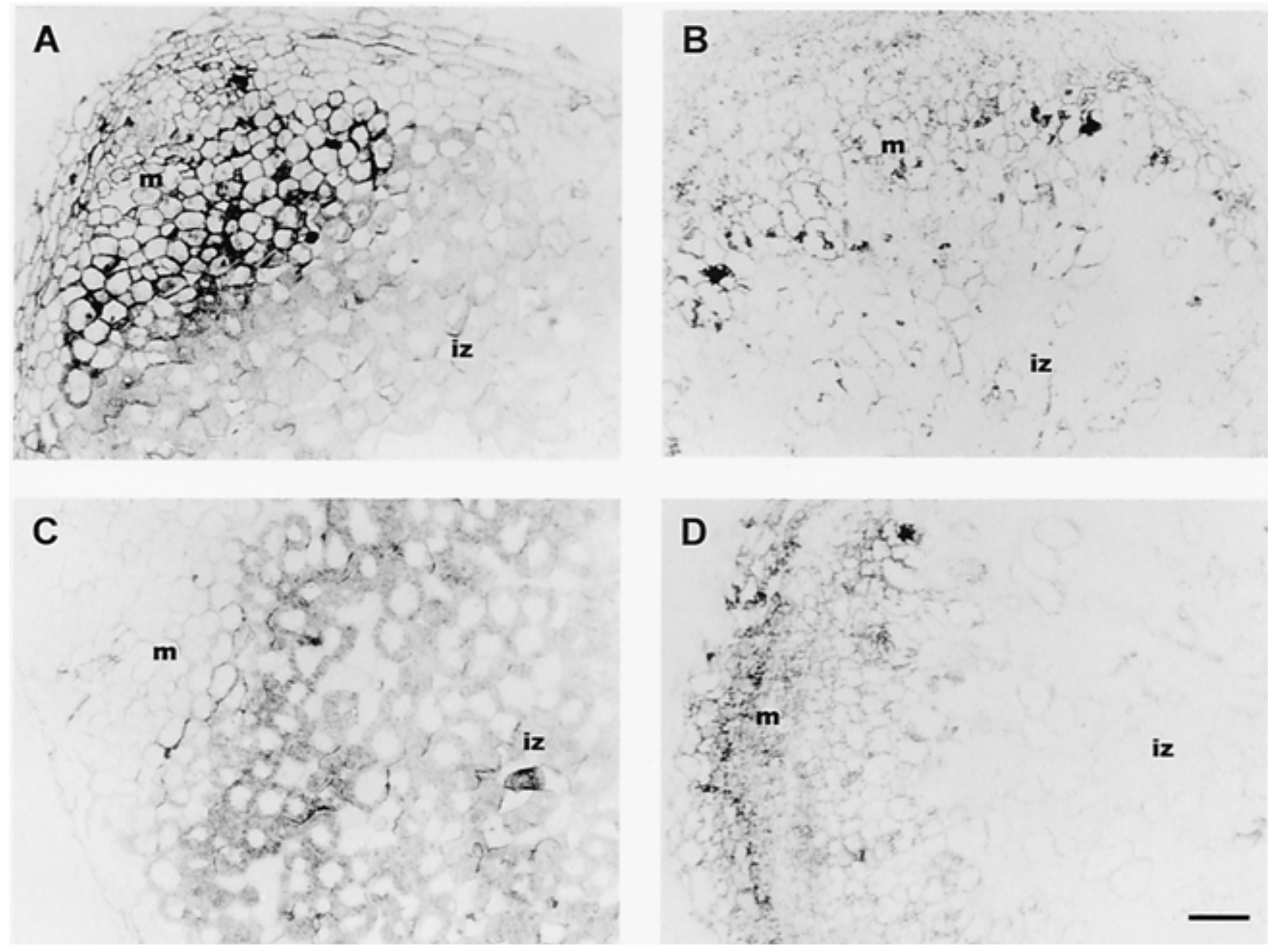

Fig. 4. Immunogold and silver enhancement on longitudinal semithin sections of 21-day-old pea nodule tissue developed in the $\mathbf{A}$ and $\mathbf{C}$, presence or $\mathbf{B}$ and D, absence of boron and probed with MAC 207 to localize A and B, peribacteroid and cell-membrane glycoproteins or C and D, MAC 206 to localize peribacteroid and membrane glycolipids antibodies. $\mathrm{m}=$ nodule meristem; iz $=$ infected zone. $\mathrm{Bar}=0.1 \mathrm{~mm}$. 
abnormal symbiosome development (Fig. 1), all three isoforms of PsNLEC-1 were detected (Fig. 3B), although most of the R76 antigens were localized in the cytoplasmic compartment of B-deficient nodules (Fig. 6C and D). This suggests that modified isoforms $1 \mathrm{~A}$ and $1 \mathrm{~B}$ are targeted abnormally, resulting in a cytoplasmic localization as reported for PsNLEC-1C in sym 31 nodules. Both observations suggest that the carbohydrate moiety of isoforms A and B may be crucial for targeting symbiosome and bacteroid maturation. All of these findings could correlate the expression of nodule lectin- like glycoproteins with signaling for the maturation of the bacteroid in indeterminate nodules.

Because free-living Rhizobium leguminosarum 3841 apparently is not affected by the absence of $B$, as judged by its LPS pattern (Fig. 2), normal growth, and exopolysaccharide production (not shown), the alterations of symbiosome maturation should result from the abnormal development of plant-derived components such as the above-reported lectinlike glycoproteins. Nevertheless, it is difficult to correlate these results with specific effects of the nutritional status of
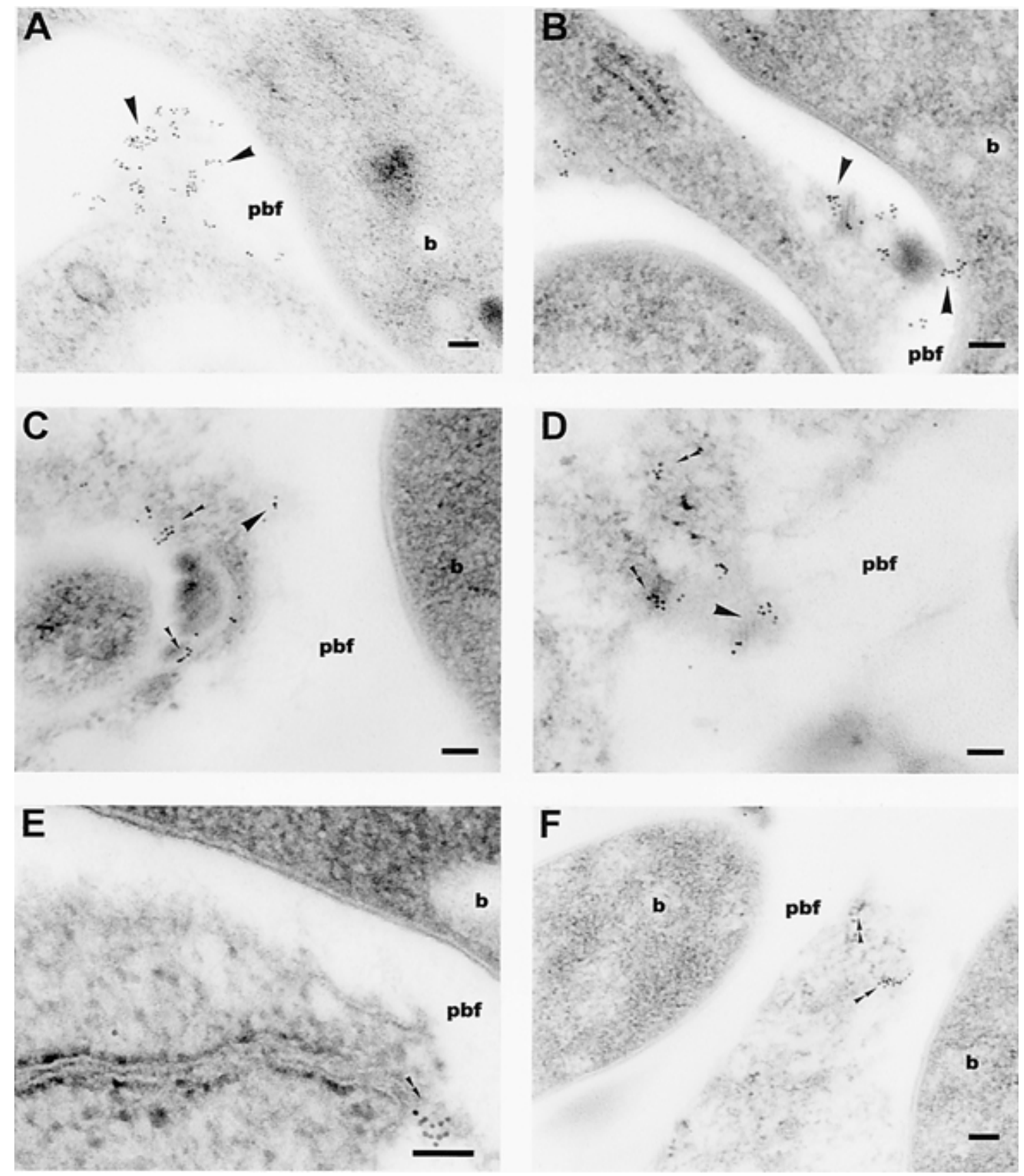

Fig. 5. Electron micrographs of nodule ultrathin sections showing the presence in infected cells of epitopes recognized by the MAC 254 antibody following immunostaining and immunogold (10 nm of gold anti-rat immunoglobulin G). A and B, Nodule from plants grown in the presence of boron. CF, Nodule from plants grown in the absence of boron. $\mathrm{b}=$ endophytic bacteria; $\mathrm{pbf}=$ peribacteroid fluid compartment. Arrowheads show labeling in the peribacteroid space. Double arrowheads indicate antigen associated with cytoplasmic membranes. Bar $=0.1 \mu \mathrm{m}$. 
the symbiosome because B is acting in many other plant physiological processes. The study of nodule development in the absence of $\mathrm{B}$, however, could help provide an understanding to its role in plant physiology, especially on membrane (i.e., vesicle targeting and transmembrane transport) and cell wall integrity. Furthermore, B deprivation affects nodule development similarly to that described for plant nodulation or ineffective Rhizobium spp. mutants. Consequently, investigating the effects of $\mathrm{B}$ in nodule development could shed new light on unclear topics of the rhizobiaplant symbiotic relationship.

\section{MATERIALS AND METHODS}

\section{Plant growth and inoculation.}

Pea (Pisum sativum cv. Argona) seeds were surface sterilized with $70 \%$ (vol/vol) ethanol for $1 \mathrm{~min}$ and $10 \%$ (vol/vol) sodium hypochlorite for $20 \mathrm{~min}$, soaked for $4 \mathrm{~h}$ in sterile distilled water, and germinated on wet filter paper at $25^{\circ} \mathrm{C}$. After 4 days, seedlings were transferred to plastic growth pots and cultivated on B-free perlite with FP medium for legumes (Fahraeus 1957).
For B-free cultures, B was removed from the micronutrients solution. For cultures with the normal content of $\mathrm{B}$, the micronutrient $\left(\mathrm{H}_{3} \mathrm{BO}_{3}\right)$ was added to a final concentration of 0.1 $\mathrm{mg}$ of $\mathrm{B}$ per liter. All solutions were prepared and stored in polyethylene containers demonstrated previously not to release boron, even under sterilizing conditions (Mateo et al. 1986). Boron was determined to be in the solutions and media prior to using them, and no boron was detected (detection limit was $0.02 \mu \mathrm{g}$ per $\mathrm{ml}$ ). B concentration was determined with Azomethine $\mathrm{H}$ at $\mathrm{pH} 5.1$ (Wolf 1974) and a Technicon (Tarrytown, NY, U.S.A.) automatic analytical system (Martinez et al. 1986). Before inoculation, pea seedlings were grown without added $\mathrm{B}$ for 15 days (including germination time) to drain the B stored in seeds.

Plants were inoculated with $1 \mathrm{ml}$ per seedling of approximately $10^{8}$ cells of $R$. leguminosarum bv. viciae 3841 per ml from an exponential culture in TY (tryptone-yeast extract) medium (Beringer 1974) and maintained in a growth cabinet at $22^{\circ} \mathrm{C}$ day- $18^{\circ} \mathrm{C}$ night temperatures, with a 16 - to 8 -h photoperiod and an irradiance of $190 \mu \mathrm{mol} \mathrm{m} \mathrm{m}^{-2}$ per s. Relative humidity was kept between 60 and $70 \%$.
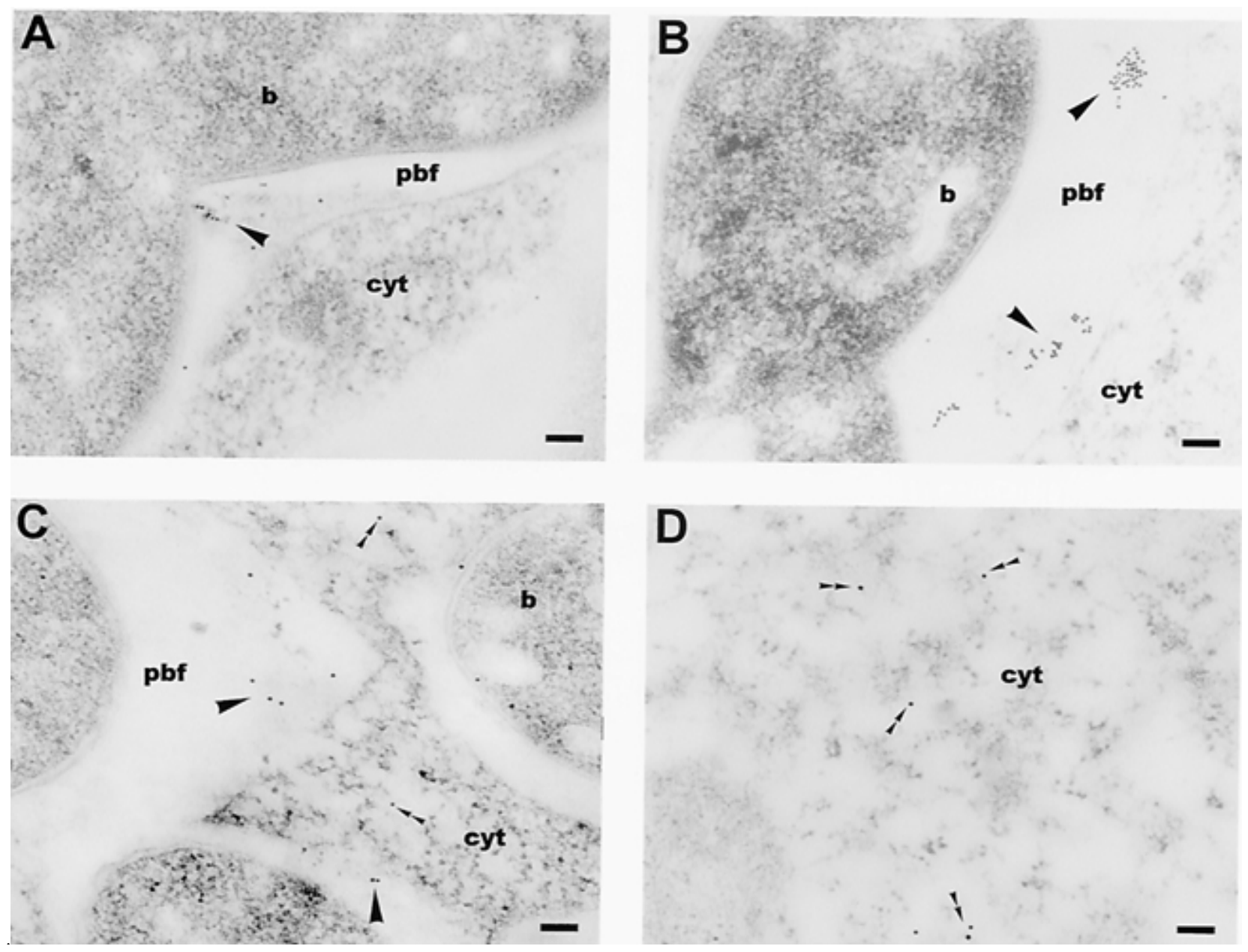

Fig. 6. Electron micrographs of nodule ultrathin sections showing the presence in infected cells of PsNLEC-1 recognized by R76 antiserum following immunostaining and immunogold (10 nm of gold anti-rat immunoglobulin G). Nodule from plants grown in the $\mathbf{A}$ and $\mathbf{B}$, presence or $\mathbf{C}$ and $\mathbf{D}$, absence of boron. $\mathrm{b}=$ endophytic bacteria; $\mathrm{pbf}=$ peribacteroid fluid compartment; cyt = cytoplasm. Arrowheads show labeling in the peribacteroid space. Double arrowheads indicate antigen associated with cytoplasmic membranes. Bar $=0.1 \mu \mathrm{m}$. 


\section{Bacterial cultures.}

To analyze the effects of B on the LPS of R. leguminosarum strain 3841 , bacteria were grown in polyethylene flasks containing liquid Y medium (Sherwood 1970) vigorously shaken in an orbital shaker for $48 \mathrm{~h}$ at $28^{\circ} \mathrm{C}$. For cultures with B, the micronutrient was added as $\mathrm{H}_{3} \mathrm{BO}_{3}$ to a final concentration of $0.1 \mathrm{mg}$ of B per liter. For B-free cultures, cells were washed seven times and recultured three times in B-free $\mathrm{Y}$ medium before inoculation. The absence of $\mathrm{B}$ in the flasks and nutrient solutions was tested, as previously indicated.

\section{Antibodies and antisera.}

The rat monoclonal antibodies used in this study have been described previously by Perotto et al. (1991). MAC 207 and 272 recognize carbohydrate components on a range of glycoproteins $\left(\mathrm{M}_{\mathrm{R}} 45\right.$ to $\left.120 \mathrm{kDa}\right)$ present in plasma and peribacteroid membrane glycocalyx. MAC 206 and 255 recognize glycolipid components of the plant and peribacteroid membrane glycocalyx. MAC 254 and 266 react with carbohydrate epitopes associated with glycoproteins that are targeted to the peribacteroid fluid (Perotto et al. 1991), including two isoforms of a nodule-specific, lectin-like glycoproteins (PsNLEC-1A, and -1B) of approximately 30 and $27 \mathrm{kDa}$ (Dahiya et al. 1997). Rabbit antiserum R76, reacting with PsNLEC-1, was prepared from antigenic material obtained by heterologous expression of the PsNlecl cDNA cloned in Escherichia coli, as described by Dahiya et al. (1997). The antiserum was precipitated routinely with $E$. coli lysate to remove cross-reacting antibodies. For immunogold staining, R76 was further purified by immunoadsorption to heterologous PsNLEC-1 protein expressed in E. coli.

\section{Fractionation of nodules.}

Pea nodules were homogenized at $4{ }^{\circ} \mathrm{C}$ in Tris-dithiothreitol buffer (50 mM Tris- $\mathrm{HCl}, \mathrm{pH} \mathrm{7.5}$; and $10 \mathrm{mM}$ dithiothreitol) containing $0.5 \mathrm{M}$ sucrose. The soluble fraction of the homogenate was collected by filtration through Miracloth (Calbiochem, Madrid, Spain) and centrifuged for $30 \mathrm{~min}$ at $100,000 \mathrm{~g}$ to remove debris. The resulting pellet contained the symbiosome and bacteroid fractions. Bacteroids still enclosed by the peribacteroid membrane were prepared by fractionation and purified by centrifugation through a sucrose gradient, as described by Brewin et al. (1985). To avoid losses of soluble glycoproteins resulting from breaks in B-deficient membranes, all of the soluble fractions derived from the supernatants obtained during fractionation were collected and centrifuged for $30 \mathrm{~min}$ at $100,000 \mathrm{~g}$. The resulting supernatant was used to study glycoproteins from the peribacteroid fluid (PBF).

\section{SDS-PAGE and immunoblotting.}

Samples for gel separation were extracted by heating in SDS buffer for $10 \mathrm{~min}$ at $100^{\circ} \mathrm{C}$. After centrifugation to remove insoluble debris, the extracts $(10 \mu \mathrm{g}$ protein loaded per lane) were subjected to $12 \%$ acrylamide minigels (Laemmli 1970). Gels were transferred electrophoretically to membranes of nitrocellulose (Bittner et al. 1980). Blots were incubated with $5 \%$ bovine serum albumin) in Tris-buffered saline (50 $\mathrm{mM}$ Tris- $\mathrm{HCl}, \mathrm{pH} 7.4$; and $200 \mathrm{mM} \mathrm{NaCl}$ ) buffer containing a monoclonal antibody (1\% hybridoma culture supernatant). Immunostaining was visualized with goat anti-rat immu- noglobulin $\mathrm{G}$ (IgG; for monoclonal antibodies) or goat antirabbit IgG (for antiserum). In both cases, an alkaline phosphatase-conjugated secondary antibody was used (Bradley et al. 1988).

For bacterial LPS studies, gels were stained after electrophoresis with Bio-Rad (Madrid, Spain) silver-staining reagents, following periodate oxidation (Wood et al. 1989).

\section{Microscopy on nodule sections.}

Pea nodules developed in the presence or absence of B were fixed overnight at $4^{\circ} \mathrm{C}$ in $2.5 \%$ (vol/vol) glutaraldehyde in 0.1 $\mathrm{M}$ sodium cacodylate buffer, $\mathrm{pH}$ 7.2. Nodules were dehydrated in an ethanol series and embedded in LR White resin. Ultrathin, silver-gold nodule sections were processed for immunogold staining following transmission electron microscopy, and semithin sections were processed for immunogold staining and silver enhancement, in accordance with methods described previously (Rae et al., 1991). A goat anti-rat IgG (to reveal monoclonal antibodies labeling) or a goat anti-rabbit IgG (to reveal R76 labeling) conjugated to colloidal gold (10 $\mathrm{nm}$ gold particles) (Amersham Pharmacia Biotech, Little Chalfont, Buckinghamshire, U.K.) was used as a secondary antibody. Controls (data not shown) without antibody to identify background staining and immunostaining with other antibodies such as MAC 265 (anti-infection thread matrix glycoprotein) or 57 (anti-R. leguminosarum 3841 LPS), shown previously to label B-deprived nodule sections (Bolaños et al. 1996), were included to demonstrate that there was no lack of staining as a result of poor fixation of B-deficient tissues.

\section{ACKNOWLEDGMENTS}

The antibodies and antisera used in this study were provided by N. J. Brewin (John Innes Centre, Norwich, U.K.). L. Bolaños received a grant from the Comunidad de Madrid. This work was supported by Programa Sectorial de Promoción General del Conocimiento (M.E.C.), PB980114-CO2-01.

\section{LITERATURE CITED}

Beringer, J. E. 1974. R factor transfer in Rhizobium leguminosarum. J. Gen. Microbiol. 84:188-198.

Bittner, M., Kupferer, P., and Morris C. F. 1980. Electrophoretic transfer of proteins and nucleic acids from the slab gels to diazobenzyloxymethyl cellulose or nitrocellulose sheets. Anal. Biochem. 102:459471.

Bolaños, L., Esteban, E., De Lorenzo, C., Fernández-Pascual, M., De Felipe, M. R., Gárate, A., and Bonilla, I. 1994. Essentiality of boron for symbiotic dinitrogen fixation in pea (Pisum sativum)-Rhizobium nodules. Plant Physiol. 104:85-90.

Bolaños, L., Brewin, N. J., and Bonilla, I. 1996. Effects of boron on Rhizobium-legume cell-surface interactions and nodule development. Plant Physiol. 110:1249-1256.

Bonilla, I., García-González, M., and Mateo, P. 1990. Boron requirement in cyanobacteria: Its possible role in the early evolution of photosynthetic organisms. Plant Physiol. 94:1554-1560.

Bonilla, I., Mergold-Villaseñor, C., Campos, M. E., Sánchez, N., Pérez, H., López, L., Castrejón, L., Sánchez, F., and Cassab, G. I. 1997. The aberrant cell walls of boron-deficient bean root nodules have no covalently bound hydroxyproline-/proline-rich proteins. Plant Physiol. 115:1329-1340.

Bradley, D. J., Wood, E. A., Larkins, A. P., Galfre, G., Buchter, G. W., and Brewin, N. J. 1988. Isolation of antibodies reacting with peribacteroid membranes and other components of pea root nodules containing Rhizobium leguminosarum. Planta 173:149-160.

Brenchley, W. E., and Thornton, H. G. 1925. The relation between the 
development, structure and functioning of the nodules on Vicia faba, as influenced by the presence or absence of boron in the nutrient medium. Proc. R. Soc. Lon. B. Sci. 98:373-398.

Brewin, N. J. 1991. Development of the legume root nodule. Annu. Rev. Cell. Biol. 7:191-226.

Brewin, N. J., Robertson, J. G., Wood, E. A., Wells, B. A., Larkins, A. P., Galfre, G., and Butcher, G. W. 1985. Monoclonal antibodies to antigens in the peribacteroid membrane from Rhizobium-induced root nodules of pea cross react with plasma membrane and Golgi bodies. EMBO J. 4:605-611.

Brewin, N. J., Bolaños, L., Dahiya, P., Gardner, C. D., Hernández, L. E., Kardailsky, I. V., Rathbun, E. A., and Sherrier, D. J. 1995. Differentiation of the symbiosome compartment in pea nodule cells. Pages 455 460 in: Nitrogen Fixation: Fundamentals and Applications. I. A. Tikhonovich, N. A. Provorov, V. E. Romanov, and W. E. Newton, eds. Kluwer Academic Publishers, Dordrecht, The Netherlands.

Dahiya, P., Kardailsky, I. V., and Brewin, N. J. 1997. Immunolocalization of PsNLEC-1, a lectin-like glycoprotein expressed in developing pea nodules. Plant Physiol. 115:1431-1442.

Dahiya, P., Sherrier, D. J., Kardailsky, I. V., Borisov, A. Y., and Brewin, N. J. 1998. Symbiotic gene Sym31 controls the presence of a lectinlike glycoprotein in the symbiosome compartment of nitrogenfixing pea nodules. Mol. Plant-Microbe Interact. 11:915-923.

Day, D. A., and Udvardi, M. K. 1993. Metabolite exchange across symbiosome membranes. Symbiosis 14:175-189.

De Maagd, R. A., Yang, W. C., Goosen-de Roo, L., Mulders, I. H. M., Roest, H. P., Spaink, H. P., Bisseling, T., and Lugtengerg, B. J. J. 1994. Down-regulation of expression of the Rhizobium leguminosarum outer membrane protein gene ropA occurs abruptly in interzone II-III of pea nodules and can be uncoupled from nif gene activation. Mol. Plant-Microbe Interact. 7:276-281.

Fahraeus, G. 1957. The infection of clover root hairs by nodule bacteria studied by a simple glass technique. J. Gen. Microbiol. 16:374-381.

Faye, L., Johnson, K. D., Storm, A., and Chrispeels, M. J. 1989. Structure, biosynthesis and function of asparagine-linked glycans on plant glycoproteins. Physiol. Plant 75:309-314.

Goldbach, H. E. 1997. A critical review of current hypotheses concerning the role of boron in higher plants: Suggestion for further research and its methodological requirements. J. Trace Microprobe Tech. 15:51-92.

Kannenberg, E. L., and Brewin, N. J. 1994. Molecular mechanisms of host plant invasion by Rhizobium. Trends Microbiol. 2:277-283.

Kannenberg, E. L., Perotto, S., Bianciotto, V., Rathbun, E. A., and Brewin, N. J. 1994. Lipopolysaccharide epitope expression of Rhizobium bacteroids as revealed by in situ immunolabelling of pea root nodule sections. J. Bacteriol. 176:2021-2032.

Kardailsky, I. V., Sherrier, D. J., and Brewin, N. J. 1996. Identification of a new pea gene, PsNlec1, encoding a lectin-like glycoprotein isolated from the symbiosomes of root nodules. Plant Physiol. 111:49-60.

Laemmli, U. K. 1970. Cleavage of structural proteins during the assembly of the head of bacteriophage T4. Nature 227:680-685.

Marschner, H. 1995. Functions of mineral nutrients: Micronutrients, boron. Pages 379-396 in: Mineral Nutrition of Higher Plants. Academic Press, London.

Martínez, F., Mateo, P., Bonilla, I., Fernández-Valiente, E., and Gárate, A. 1986. Growth of Anacystis nidulans in relation to boron supply. Isr. J. Bot. 35:17-21.

Mateo, P., Bonilla, I., Fernández-Valiente, E., and Sánchez-Maeso, E. 1986. Essentiality of boron for dinitrogen fixation in Anabaena sp. PCC 7119. Plant Physiol. 81:430-433.

Mazurek, M., and Perlin, A. S. 1963. Borate complexing by fivemembered-ring vic-diols: Vapor observations. Can. J. Chem. 41:24032411.
Mellor, R. B. 1989. Bacteroids in the Rhizobium-legume symbiosis inhabit a plant internal lytic compartment: Implications for other microbial endosymbioses. J. Exp. Bot. 40:831-839.

Miao, G. H., Hong, Z., and Verma, D. P. S. 1992. Topology and phosphorylation of soybean nodulin-26, an intrinsic protein of the peribacteroid membrane. J. Cell. Biol. 118:481-490.

O'Neill, M. A., Warrenfeltz, D., Kates, K., Pellerin, P., Doco, T., Darvill, A. G., and Albersheim, P. 1996. Rhamnogalacturan-II, a pectic polysaccharide in the walls of growing plant cell, forms a dimer that is covalently cross-linked by a borate ester. In vitro conditions for the formation and hydrolysis of the dimer. J. Biol. Chem. 271:22923-22930.

Parr, A. J., and Loughman, B. C. 1983. Boron and membrane function in plants. Pages 87-107 in: Metals and Micronutrients: Uptake and Utilization by Plants. D. A. Robb and W. S. Pierpoint, eds. Academic Press, London.

Perotto, S., VandenBosch, K. A., Butcher, G. W., and Brewin, N. J. 1991. Molecular composition and development of the plant glycocalyx associated with the peribacteroid membrane of pea root nodules. Development 112:763-773

Perotto, S., Donovan, N., Drøbak, B. J., and Brewin, N. J. 1995. Differential expression of a glycosyl inositol phospholipid antigen on the peribacteroid membrane during pea nodule development. Mol. PlantMicrobe Interact. 8:560-568

Rae, A. L., Perotto, S., Knox, J. P., Kannenberg, E. L., and Brewin, N. J. 1991. Expression of extracellular glycoproteins in the uninfected cells of developing pea nodule tissue. Mol. Plant-Microbe Interact. 4:563-570.

Romanov, V. I., Gordon, A. J., Minchin, F. R., Witty, J. F., Skot, L., James, C. L., Borisov, A. Y., and Tikhonovich, I. A. 1995. Anatomy, physiology and biochemistry of root nodules of Sprint-2Fix(-), a symbiotically defective mutant of pea (Pisum sativum L.). J. Exp. Bot. 46:1809-1816.

Sherrier, D. J., Borisov, A. Y., Tikhonovich, I. A., and Brewin, N. J. 1997. Immunocytological evidence for abnormal symbiosome development in nodules of the pea mutant line Sprint2Fix ${ }^{-}$(sym31). Protoplasma 199:57-68.

Sherwood, M. T. 1970. Improved synthetic medium for the growth of Rhizobium. J. Appl. Bacteriol. 33:708-713.

Sindhu, S. S., Brewin, N. J., and Kannenberg E. L. 1990. Immunochemical analysis of lipopolysaccharides from free-living and endosymbiotic forms of Rhizobium leguminosarum. J. Bacteriol. 172:1804-1813.

Smyth, D. A., and Dugger, W. M. 1981. Cellular changes during boron deficient culture of the diatom Cylindrotheca fusiformis. Plant Physiol. 51:111-117.

Tikhonovich, I. A., Borisov, A. Y., Chvabauskene, I. A., Kamardin, N. N., Kravchenko, L. V., Lebsky, V. K., Minchin, F., Morzhina, E. V., Romanov, V. I., Skot, L., Tchetkova, S. A., and Tsyganov, V. E. 1995. Plant genetic control at the crucial steps of symbiosis. Pages 461-466 in: Nitrogen Fixation: Fundamentals and Applications. I. A. Tikhonovich, N. A. Provorov, V. E. Romanov, and W. E. Newton, eds. Kluwer Academic Publishers, Dordrecht, The Netherlands.

VandenBosch, K. A., Rodgers, L. R., Sherrier, D. J., and Kishinevsky, B. D. 1994. A peanut nodule lectin in infected cells and in vacuoles and the extracellular matrix of nodule parenchyma. Plant Physiol. 104:327-337.

Verma, D. P. S. 1992. Signals in root nodule organogenesis and endocytosis of Rhizobium. Plant Cell 4:373-382.

Wolf, B. 1974. Improvement in the Azomethine H method for determination of boron. Commun. Soil Sci. Plant Anal. 5:39-44.

Wood, E. A., Butcher, G. W., Brewin, N. J., and Kannenberg, E. L. 1989. Genetic derepression of a developmentally regulated lipopolysaccharide antigen from Rhizobium leguminosarum 3841. J. Bacteriol. 171:4549-4555. 